\title{
High-Resolution UV Spectroscopy of Molecular Complexes
}

\author{
H. J. Neusser, R. Sußmann, A. M. Smith, E. Riedle, and Th. Weber
}

Institut für Physikalische und Theoretische Chemie, Technische Universität München, Lichtenbergstraße 4, W-8046 Garching, Germany

\section{Clusters / Molecular Beams / Molecular Interactions / Molecular Structure / Spectroscopy, Ultraviolet}

Information on the structure, the rigidity and the intermolecular potential of molecular complexes is essential to our understanding of the physical and chemical properties of molecular complexes. In this work we would like to demonstrate that rotationally resolved UV spectroscopy provides precise new information on these topics. In particular, the structure and van der Waals bond length of benzene-X complexes $\left(\mathrm{X}=\mathrm{Ne}, \mathrm{Ar}, \mathrm{Kr}, \mathrm{Xe}, \mathrm{N}_{2}\right.$ ) have been experimentally determined. In the benzene- $\mathrm{N}_{2}$ complex with its parallel-stacked configuration, the two constituents, benzene and $\mathrm{N}_{2}$, can rotate against each other. Rotationally resolved vibronic van der Waals bands allow the clear assignment of the excited van der Waals vibrations. Their anharmonicity and the vibrationally averaged rotational constants provide basic information on the intermolecular potential. For the example of the benzene-Ar $\mathrm{Ar}_{2}$ trimer it is shown that isomeric structures exist that are rigid on the nanosecond time scale.

\section{Introduction}

Interest in the UV spectroscopy of van der Waals complexes has grown immensely in the last decade. This can be attributed to the convenient method of producing isolated gas phase molecular clusters in cooled supersonic beams $[1,2]$. It is the pertinent goal of these studies to obtain a microscopic understanding of van der Waals bonding intrinsic to solvation processes [3]. This goal necessitates in- 
vestigation of the structure and intramolecular dynamics of the complexes, i. e. isomerization and dissociation processes.

Up to now, nearly all investigations have been performed with relatively low spectral resolution leading to the separation of individual vibronic bands, however, with unresolved rotational structure. Thus the main source of information has been the shift of the vibronic transitions to the first electronic excited state due to complexation [4-6] and the width of the vibronic bands $[7,8]$. In these experiments it has been impossible to selectively excite individual rovibronic quantum states of these complexes that would lead to unambiguous information on the structure and dynamic processes. For this reason it is highly desirable to apply the techniques of rotationally resolved spectroscopy to complex studies.

To resolve individual rotational lines in large polyatomic systems the Doppler broadening has to be eliminated since the line spacing is so small that individual lines are hidden under the Doppler width [9]. The cold molecular beam provides an important advantage for high resolution spectroscopy, i.e. the reduction of the transversal velocity destribution and consequently of Doppler-broadening $[10-13]$. In supersonic beam expansions it is not possible to produce a single complex species but rather, in addition to the monomer constituents of the gas mixture complexes of various compositions and size are produced. The spectra of the different species may overlap and it is often difficult to disentangle them in the fluorescence excitation spectra. For this reason we combined high resolution Doppler-free excitation with mass-selective resonance-enhanced two-photon ionization [14]. The produced ions can be mass-analyzed and integrated for a selected mass. This leads to highly resolved $(\Delta v=120 \mathrm{MHz})$ mass-selected two-photon ionization spectra reflecting the intermediate state spectrum of the selected complex.

In this article we would like to demonstrate the new information that is accessible by high resolution. It is shown for the prototype complexes of benzene with noble gases and small molecules that rotationally resolved UV spectroscopy provides clear information on current issues concerning the microscopic models of these complexes. Among these are the structure of the complexes, their van der Waals bond lengths, their rigidity, and internal rotation. In particular, rotationally resolved spectra of van der Waals vibronic bands allow the unambiguous assignment of these bands. In this way fundamental information on the depth and the shape of the van der Waals potential is accessible. Finally, it can be checked by rotationally resolved spectroscopy whether rapid isomerization or a dissociation process takes place, in other words, whether the complex is stable on the nanosecond time scale of the experiment.

\section{Experimental}

In Fig. 1 the experimental setup for high resolution spectroscopy of van der Waals complexes is shown. Benzene-noble gas clusters are produced in a supersonic molecular beam [14]. Benzene at a vapour pressure of 40 Torr is seeded in $\mathrm{Ar}$ or other noble gases or mixtures of noble gases. Then the gas mixture is expanded through a small orifice, ca. $0.3 \mathrm{~mm}$ in diameter, into the first vacuum chamber. The gas throughput is limited by the short pulsed opening of a valve, which proceeds synchronously with the arrival of the laser light pulses. Through the cooling in the expansion, various homoand heteroclusters of benzene and the noble gas are formed. A conical-shaped skimmer peels away the outer shell of the molecular beam, enabling the expansion of the central part into a second vacuum chamber with a lower background pressure where the interaction with the light occurs. Thereby the Doppler broadening amounting to ca. $0.05 \mathrm{~cm}^{-1}$ in a bulk gas is reduced to some 40 $\mathrm{MHz}$ or $0.001 \mathrm{~cm}^{-1}$.

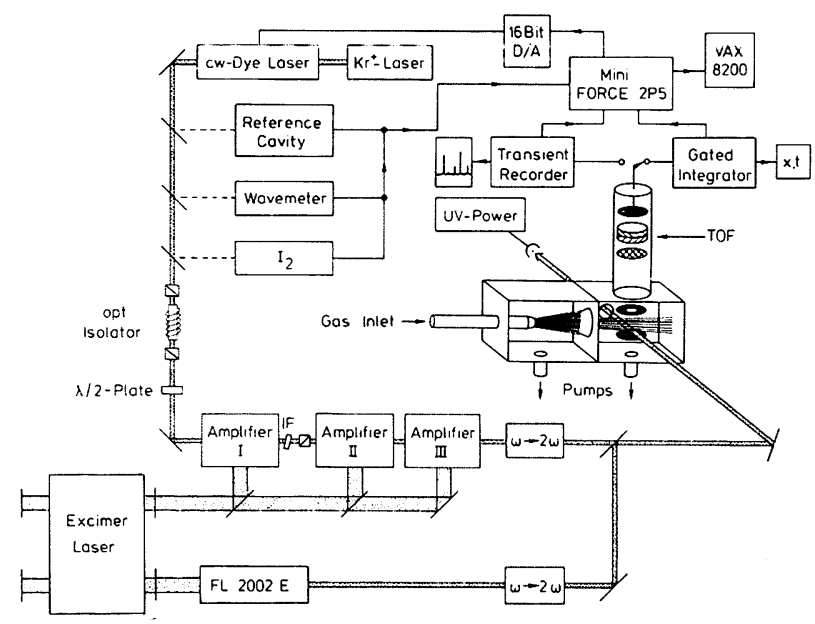

Fig. 1

Experimental setup for the recording of rotationally resolved Doppler-free UV spectra of molecular complexes by mass-selected resonance-enhanced two-photon ionization (from Ref. [14])

The narrowband light of a cw-single-mode ring dye laser around $5200 \AA$ is pulsed-amplified to a high peak power in a three-stage excimer-laser-pumped amplifier setup. The generated visible green light pulses of 10 ns length are Fourier-transform-limited with a frequency width of $80 \mathrm{MHz}$. The high peak power of $1 \mathrm{MW}$ allows efficient frequency-doubling of the green light into the ultraviolet spectral region, needed for the excitation of the $S_{1} \leftarrow S_{0}$ transition in the benzene-noble gas complexes through the absorption of one photon. The subsequent absorption of a UV photon stemming from a relatively broadband dye laser, simultaneously pumped by the excimer laser, brings the excited (to $S_{1}$ state) complexes into the ionization continuum. The ions are mass-selected and detected in a simple home-built time-of-flight mass spectrometer with a fieldfree drift length of $20 \mathrm{~cm}$ and a resulting mass resolution of about 250 . Thus the spectra of different clusters can be measured simultaneously by integrating these mass peaks with gated integrators.

\section{Benzene-Noble Gas Dimers: Structure, Van der Waals Bond Length and Dynamical Behaviour}

\section{Rotationally Resolved Spectra}

First we would like to discuss the simplest complexes consisting of a noble gas atom attached to the benzene surface. In Fig. 2 the spectrum of the $6_{0}^{1}$ band of benzene-Ar is shown when measured under a spectral resolution of 0.5 $\mathrm{cm}^{-1}$ (upper trace, strongest band). This resolution permits the separation of the different vibronic bands, however, no rotational structure is observed. A similar spectrum was first obtained by Fung et al. [15] and Menapace et al. [16]. In the lower trace the same band is presented with a resolution 
of $120 \mathrm{MHz}$ achieved in our previous work [14]. It displays the rotational structure of a prolate symmetric top with most of the rotational lines resolved. The well resolved strong lines at the low and high energy side of the spectrum are assigned to the $P$ and $R$ branch, respectively. The 7 strong features in the center of the bond are subbranches of the Q-branch with partly overlapping rotational lines.

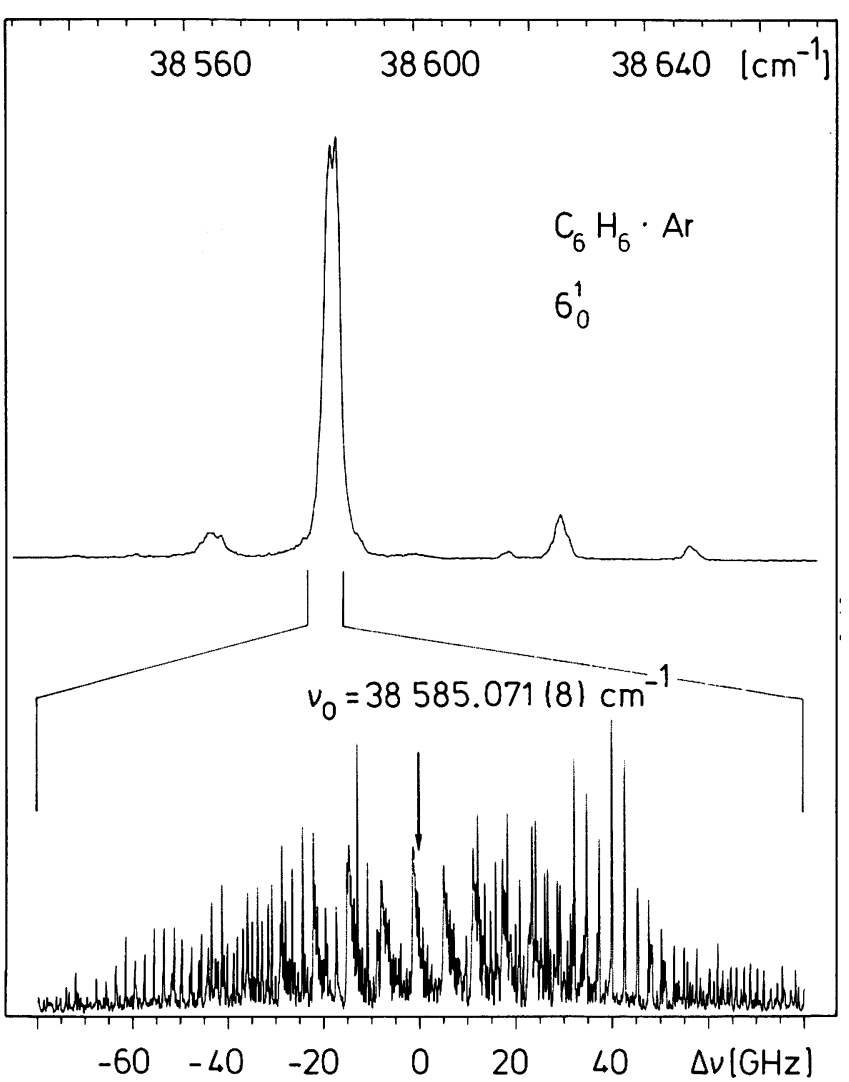

Fig. 2

Mass-selected resonance-enhanced two-photon ionization spectra of the $6_{0}^{1}$ band of $\mathrm{C}_{6} \mathrm{H}_{6} \cdot \mathrm{Ar}$ under different resolution. Upper trace: low resolution measurement. Lower trace: rotationally resolved spectrum recorded with the experimental setup shown in Fig. 1

For a precise determination of the rotational constants a two stage computer fit to the line positions was performed according to a symmetric top energy formula. In the first step, the ground state constant $B_{0}^{\prime \prime}$ is evaluated by combination differences $\left(B_{0}^{\prime \prime}=0.039402576(4) \mathrm{cm}^{-1}\right)$, i.e. frequency differences of transitions starting at different ground states but leading to the same excited state. In a second step the excited state constants $A_{\mathrm{v}}^{\prime}=0.090866(3) \mathrm{cm}^{-1}, B_{\mathrm{v}}^{\prime}=$ $0.040090(2) \mathrm{cm}^{-1}$ are fitted to about 230 unblended rotational lines. The standard deviation of the fit is $29.6 \mathrm{MHz}$ which is less than a quarter of the spectral linewidth. By means of a simultaneously measured iodine spectrum it is possible to fix the band origin $v_{0}$ to an accuracy of 0.008 $\mathrm{cm}^{-1}$. In this way the red shift of the cluster transition from the respective transition in neat benzene can be determined with high precision. The quality of the fit is demonstrated in Fig. 3. Here the central part of the band is shown on an extended scale. The upper trace gives the experimental spectrum; the lower trace shows the calculated spectrum for comparison. From the good agreement and the absence of asymmetry splitting of the indicated lines with high $J$ and low $K$ quantum number it is concluded that the benzeneAr van der Waals complex is a symmetric top.

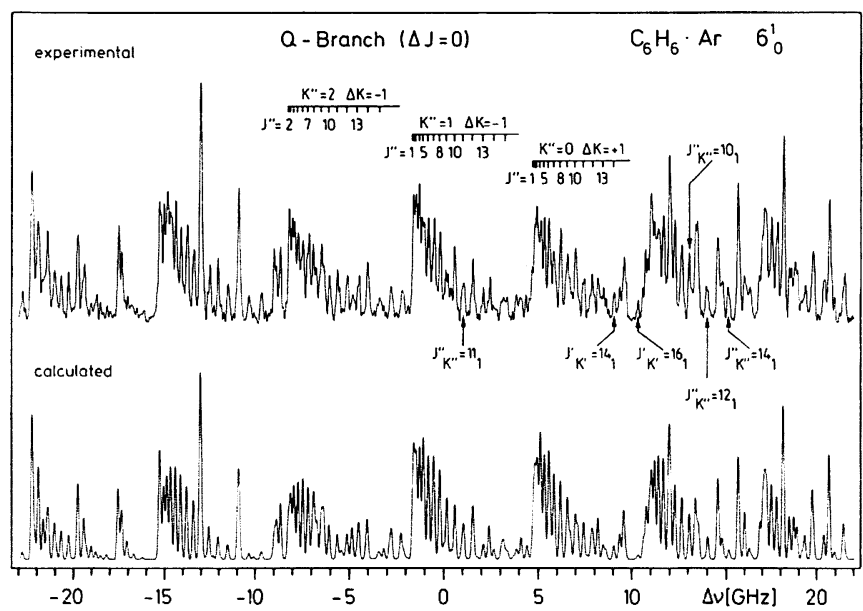

Fig. 3

Central part (Q-branch) of the $6_{0}^{1}$ band of $\mathrm{C}_{6} \mathrm{H}_{6} \cdot \mathrm{Ar}$. Upper trace: experimental spectrum. Lower trace: spectrum calculated from the rotational constants obtained from the analysis of the experimental spectrum. Several rovibronic lines with either $K^{\prime \prime}=1$ or $K^{\prime}=1$ and high $J$ are marked by arrows. These lines should display a splitting if the complex structure deviates from a symmetric top (from Ref. [14])

\section{Structure}

To be a symmetric top the Ar atom of the benzene-Ar complex has to be located above the benzene ring on the $\mathrm{C}_{6}$ rotational axis. From the vibrationally averaged rotational constants $B_{0}^{\prime \prime}$ and $B_{v}^{\prime}$, accurate values for the average bond distance $\left\langle r_{\mathrm{Ar}}\right\rangle$ in the $\mathrm{S}_{0}$ and the $\mathrm{S}_{1}$ state are calculated. We find a decrease of the van der Waals distance from $3.58 \AA$ in the $\mathrm{S}_{0}$ state to $3.52 \AA$ in the electronically excited $\mathrm{S}_{1}$ state. This can be explained by a higher polarizability of benzene in the $S_{1}$ state causing a stronger attractive force. In addition, the ring size of benzene increases after electronic excitation, so that the Ar atom can come closer to the benzene ring. Similar experiments have been performed for other benzenenoble gas $(X)$ complexes $(X=N e, A r, K r, X e)[14,17]$. The results for the bandshift and the average van der Waals bond

Table 1

Experimentally determined averaged van der Waals bond lengths $\left\langle r_{\mathrm{X}}\right\rangle$ in the ground $\left(\mathrm{S}_{0}\right)$ and excited electronic state $\left(\mathrm{S}_{1}\right)$ for different benzene-noble gas dimers. $\delta v_{0}$ is the frequency shift of the $6_{0}^{1}$ band of the respective complex from the same transition in bare benzene

\begin{tabular}{lccccr}
\hline $\mathrm{C}_{6} \mathrm{H}_{6} \cdot \mathrm{X}$ & ${ }^{20} \mathrm{Ne}$ & ${ }^{40} \mathrm{Ar}$ & ${ }^{84} \mathrm{Kr}$ & ${ }^{129} \mathrm{Xe}$ \\
\hline$\left\langle r_{\mathrm{x}}\right\rangle$ & $\mathrm{S}_{0}$ & $3.460(1)$ & $3.581(1)$ & $3.676(1)$ & $3.814(20)$ \\
& $\mathrm{S}_{1}$ & $3.420(3)$ & $3.521(2)$ & $3.607(1)$ & $3.735(22)$ \\
$\delta v_{0}$ & & $-2.866(16)$ & $-21.018(16)$ & $-33.397(15)$ & $-53.666(20)$
\end{tabular}


lengths are plotted in Table 1 . The moderate increase in van der Waals bond distance is caused by the increase in atomic radius with increasing mass number down the row of noble gases. This effect is partially compensated by the strong increase in polarizability and hence attractive interaction from $\mathrm{Ne}$ to Xe. The increase of the red shift depends linearly on the rare gas polarizability which is in line with theoretical predictions [18] neglecting the differences of the ionization potentials and the van der Waals distance of the different complexes.

\section{Dynamics}

In addition to the structural data described above there is also inherent dynamic information in the rotationally resolved spectra, e.g. shown in Fig. 2. The measured linewidth in Fig. 2 corresponds to the experimental linewidth of 120 $\mathrm{MHz}$. There is no additional broadening of the lines due to fast intramolecular dynamical processes. The same result is found for the $6{ }_{0}^{1} 1_{0}^{1}$ band of the benzene-Ar complex with a vibrational excess energy of $1444 \mathrm{~cm}^{-1}$ (see Fig. 4). Thus it is clear that there are neither fast migration processes of the Ar atom along the benzene surface nor a fast dissociation of the complex. The measured complex is stable and displays a rigid structure on the nanosecond time scale of the experiment even for a vibrational energy stored in the benzene ring that is several times the binding energy of the complex (see below).

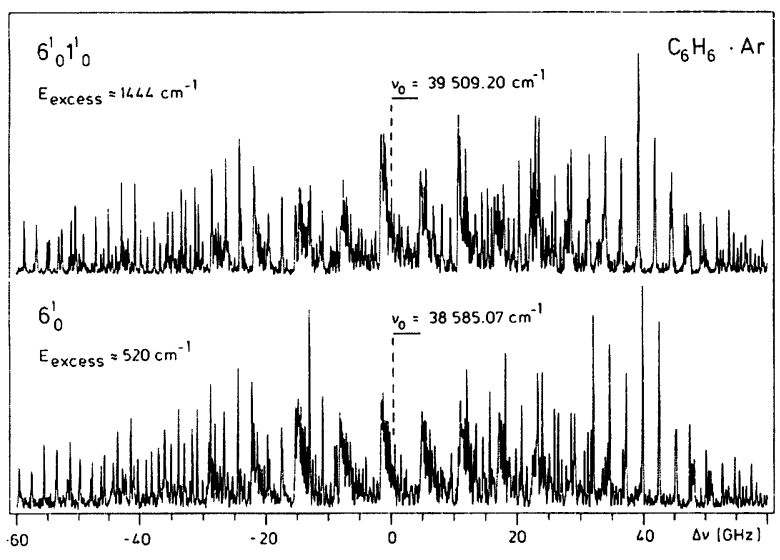

Fig. 4

Rotationally resolved UV spectra of the $6_{0}^{1}$ and $6_{0}^{1} 1_{0}^{1}$ vibronic bands of $\mathrm{C}_{6} \mathrm{H}_{6} \cdot \mathrm{Ar}$ at different excess energies. No broadening of the lines in the $6_{0}^{1} 1_{0}^{1}$ band due to fast dynamic processes is recognized

\section{Van der Waals Vibrations}

In addition to the main $6_{0}^{1}$ band additional weaker peaks on its blue side are seen in Fig. 2. Without additional information an assignment of these peaks in the low resolution spectrum by intensity arguments alone is difficult and failure-prone. Rotational structure can provide the necessary information. Recently we succeeded in measuring rotationally resolved spectra of all additional weak bands shown in Fig. $2[19,20]$. This led to the clear assignment of these bands. In Fig. 5 the rotationally resolved spectrum of one of the bands is shown for demonstration. The rotational structure of this band is similar to that of the main band shown in Fig. 2. However, the slightly changed rotational constants lead to a compression of the Q-subbranches that are blue-shaded in the $6{ }_{0}^{1}$ band (see Figs. 2 and 4) and thus to the appearance of sharp peaks in the center of the vibronic van der Waals band. From this it is clear that the additionally excited van der Waals vibration is totally symmetric and thus the stretching vibration. The frequency of the first quantum of the stretching vibration is $40.10 \mathrm{~cm}^{-1}$. Comparing the rotational structure of the remaining small peaks in the upper traces of Figs. 2 and 5 we found a strong anharmonicity for the van der Waals stretching vibration indicating a low dissociation energy of less than $150 \mathrm{~cm}^{-1}$ for the van der Waals bond. This is considerably smaller than theoretical values obtained from empirical atom-atom potentials [21 - 24] and from ab initio calculations [25]. It is, however, corroborated by our recent experimental result of $140 \mathrm{~cm}^{-1}$ obtained with a different mass spectrometric technique [26]: From the breakdown of the metastable intensity the appearance potential for the dissociation of the ionized benzene-Ar complex was determined. This value and the ionization potential of benzene lead to the dissociation energy of the neutral complex. It is interesting to discuss this result for the dissociation energy in terms of the sharp rotational line spectra obtained for the $6_{0}^{1} 1_{0}^{1}$ band (see Fig. 4)

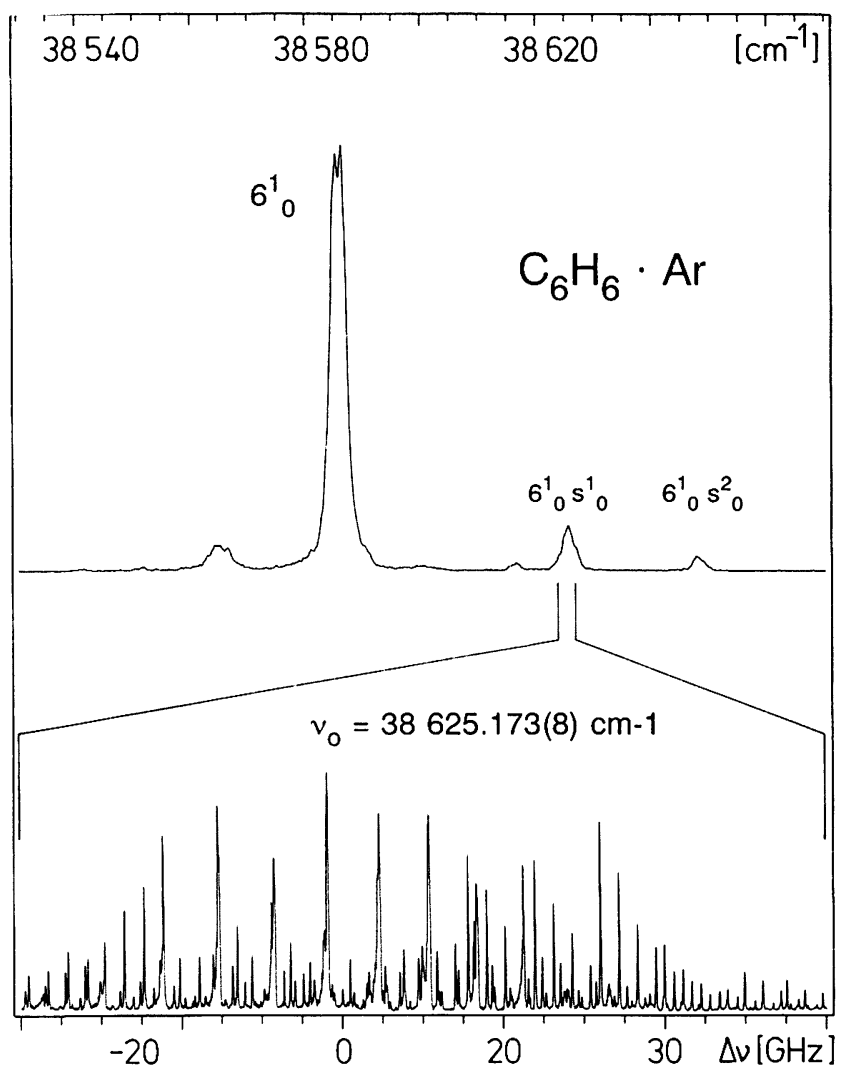

Fig. 5

Upper trace: low resolution spectrum of the $6{ }_{0}^{1}$ band of $\mathrm{C}_{6} \mathrm{H}_{6} \cdot \mathrm{Ar}$ and several smaller van der Waals vibronic bands. Lower trace: the rotationally resolved UV spectrum of the van der Waals band assigned as the $6_{0}^{1} \mathrm{~s}_{0}^{1}$ band 
leading to a final state with $1444 \mathrm{~cm}^{-1}$ vibrational excess energy, i.e. nearly ten times the binding energy of the complex. This clearly demonstrates that there is a slow energy transfer between the molecular modes and the van der Waals modes leading to a stable complex on the nanosecond time scale even at an vibrational excess energy of $1444 \mathrm{~cm}^{-1}$.

\section{Benzene-Noble Gas Trimers: Isomeric Structures}

After the structure of the benzene-Ar complex has been explored a natural question concerns the preferred position of attachment of a further noble gas atom. Here the problem arises that the inertial moments become larger and consequently the rotational lines in the spectrum denser, making their resolution more difficult. Furthermore it is not apriori clear whether the additional noble gas atoms are so strongly bound that fast isomerization processes leading to broadened spectra might be excluded at the vibrational temperature achieved in the molecular beam.

Our initial experimental results show that the benzene$\mathrm{Ar}_{2}$ complex displays a sharp rotationally resolved spectrum of the vibronic band shifted $41.86 \mathrm{~cm}^{-1}$ to the red of the bare benzene transition [27]. In Fig. 6 a recent result for the perdeuterated benzene- $\mathrm{Ar}_{2}$ complex is given. The spectrum has been measured integrating the ion current at $164 \mathrm{amu}$. i.e. the mass of $C_{6} D_{6} \cdot A_{2}$. The shape of the spectrum is similar to the one shown in Figs. 2 and 5 with many individual rotational lines resolved. As found for the protonated benzene- $\mathrm{Ar}_{2}$ complex the spectrum in Fig. 6 is a symmetric top spectrum. The structure of the complex deduced from the spectrum is illustrated in Fig. 7. Both Ar atoms lie on the $\mathrm{C}_{6}$ axis on either side of the benzene plane each at a distance of $3.58 \AA$ (1,1 structure). This bond distance agrees exactly with that in benzene-Ar (see Section III). From this we conclude that the interaction between the two noble gas atoms is effectively shielded by the aromatic ring and threebody interaction is not of importance in this configuration. From the sharpness of the rotational lines it is clear that there are no isomerization processes faster than nanoseconds and the complex displays a rigid structure. Sharp lines have been also observed for the $6_{0}^{1} \mathrm{~s}_{0}^{1}$ band of the protonated

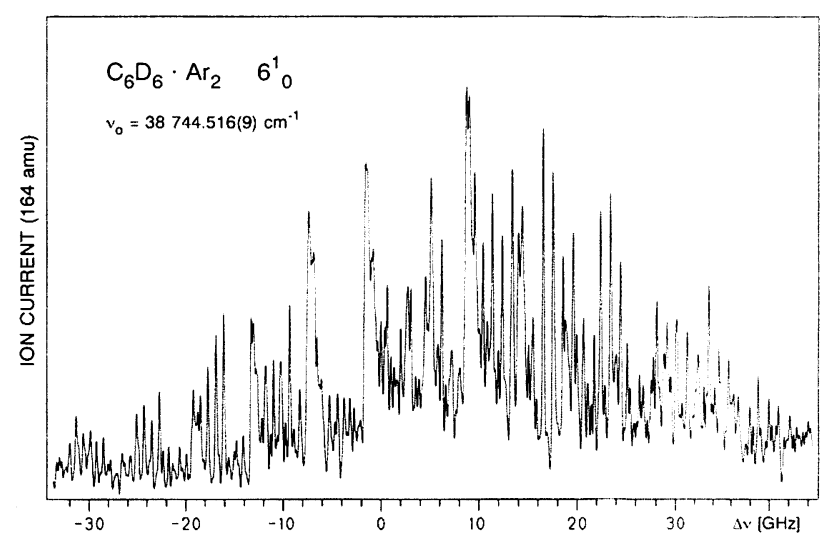

Fig. 6

Mass-selected resonance-enhanced two-photon ionization spectrum of the $6_{0}^{1}$ band of the $\mathrm{C}_{6} \mathrm{D}_{6} \cdot \mathrm{Ar}_{2}$ complex under rotational resolution benzene- $\mathrm{Ar}_{2}$ complex with one excited quantum of the stretching vibration in the final state [27]. This leads to the same conclusion that isomerization and dissociation processes are absent in this excited $\left(6^{1} s^{1}\right)$ state.

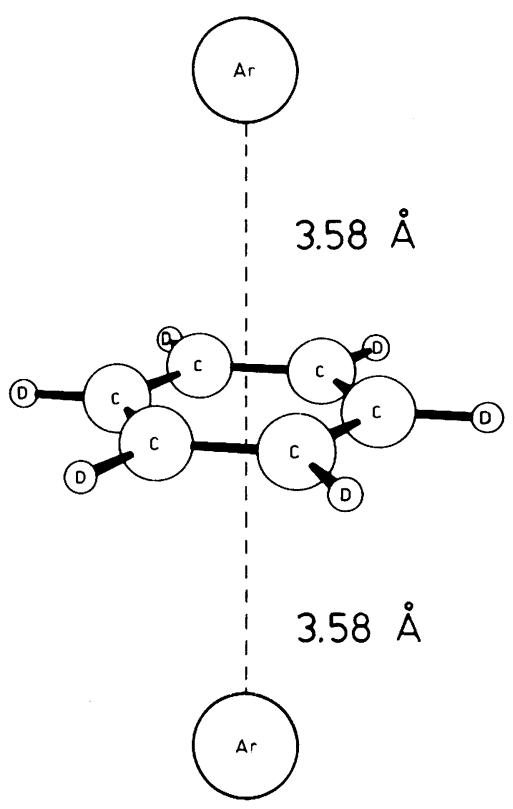

Fig. 7

Experimentally determined structure of the perdeuterated benzene$\mathrm{Ar}_{2}$ complex

Here we must ask whether the $(1,1)$ structure (see Fig. 7) is the only structure of the benzene- $\mathrm{Ar}_{2}$ complex existing in the molecular beam. In fact, recent experiments with lower (vibrational) resolution point to the existence of an additional structural isomer with both Ar atoms on the same side of the benzene ring $(2,0)$. This has been concluded from the different shift of the ionization potential, from the vibronic red shift in the $\mathrm{S}_{1} \leftarrow \mathrm{S}_{0}$ spectrum of both isomers [28], and from a rotational contour analysis [29]. In a recent theoretical work [30] it was proposed that the $(2,0)$ isomer is becoming nonrigid above $5 \mathrm{~K}$, i. e. at temperatures smaller than usually obtained in molecular beams. In contrast, the $(1,1)$ isomer should be rigid up to $50 \mathrm{~K}$. It is hardly possible to check these predictions by low resolution spectroscopy with vibrational resolution in an unambiguous way. However, through rotationally resolved spectroscopy clear statements concerning the structure and the rigidity of the investigated complexes can be made. The sharp spectrum in Fig. 6 can only originate from a complex that is stable on the nanosecond time scale. Any picosecond isomerization processes would lead to a broadening of the rotational lines. This result is in line with the theoretical predictions of Ref. [30]. In very recent experiments we have been able to resolve the rotational structure of the $6_{0}^{1}$ band of the proposed $(2,0)$ complex. The appearance of sharp rotational lines clearly demonstrates that there exists a sufficient number of $(2,0)$ complexes that are rigid on the nanosecond time scale of the experiment. It is hard to believe that the vibrational temperature of the complex is below the 
limit of $5 \mathrm{~K}$ for which a rigid structure has been predicted. Such low vibrational temperature would not be in line with the rotational temperature of a few $\mathrm{K}$ deduced from the rotational structure of the bands.

\section{Benzene-Diatomic Complexes: Benzene- $\mathrm{N}_{2}$}

Among the class of aromatic molecular complexes, benzene-diatom dimers represent the simplest complexes for which the angular momentum of the solvent molecule (the diatom) may be of importance. With their likely parallelstacked structures and low sixfold barriers to internal rotation, these complexes furthermore represent a step away from rigidity. What we clearly have learned from microwave spectroscopy is that a rotationally resolved spectrum is not only the most powerful means of structure determination, but rather the best thermometer of internal rotation. Up to now, microwave studies have been restricted to complexes with a moderately strong permanent dipole moment such as the benzene- $\mathrm{SO}_{2}$ complexes [31]. Electronic spectroscopy, on the other hand, is applicable to complexes with no dipole moment, such as the complexes studied here and, with rotational resolution, can reveal structure and dynamical information for both the ground and excited vibronic states.

We have recorded the first rotationally resolved spectrum of the benzene- $\mathrm{N}_{2}$ complex [32]. Using our constants for the ground state, the Fourier-transform microwave spec-

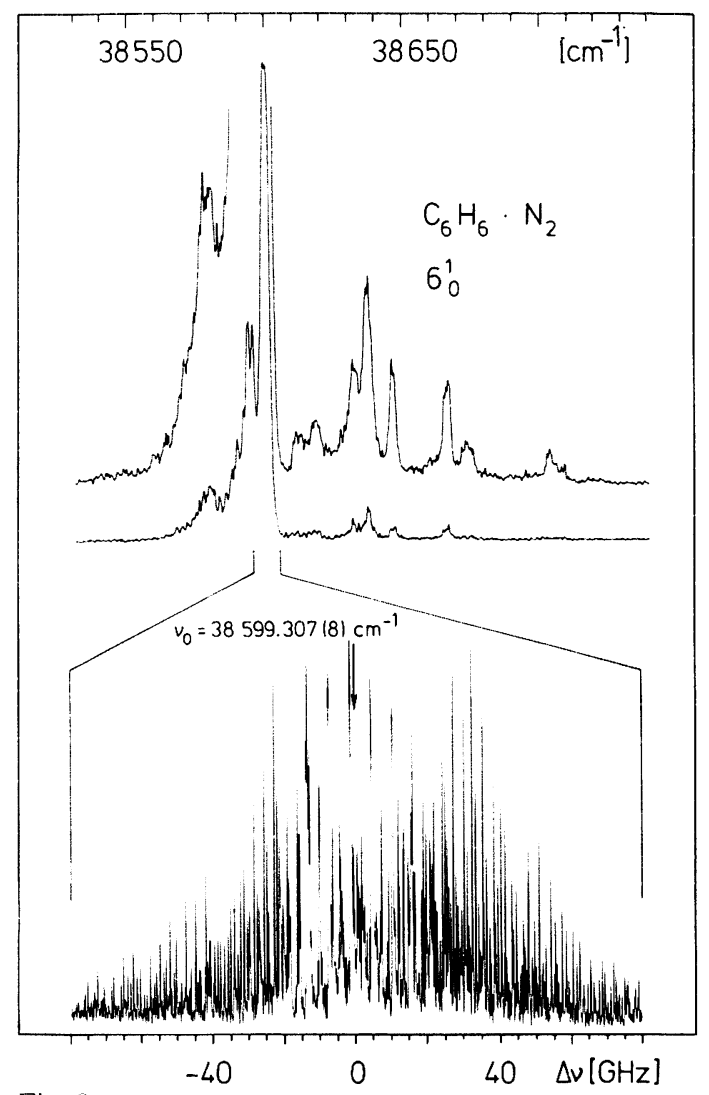

Fig. 8

Upper trace: Low resolution spectrum in the frequency range of the $6{ }_{0}^{1}$ band of the $\mathrm{C}_{6} \mathrm{H}_{6} \cdot \mathrm{N}_{2}$ complex. Lower trace: the rotationally resolved structure of the main band trum of the benzene- ${ }^{15} \mathrm{~N}_{2}$ complex has been recently located, made possible by its very small induced dipole moment [33].

With the large number of additional transitions in the vibronic spectrum, as shown at the top of Fig. 8, in this case the $6_{0}^{1}$ region, one has little chance of correctly assigning these modes with the scanty information available from a vibrationally resolved spectrum, as attempted by Novak et al. [34]. We have measured the most intense transition, i.e. the allowed $6_{0}^{1}$ band of benzene in benzene- $N_{2}$, at rotational resolution, as displayed at the bottom of Fig. 8. It is redshifted by $6.78 \mathrm{~cm}^{-1}$ from the $6_{0}^{1}$ transition in bare benzene, a value lying between that of $\mathrm{Ne}$ and $\mathrm{Ar}$, as expected from $\mathrm{N}_{2}$ 's polarizability. The rotational structure in this band, as well as that in the $6_{0}^{1} 1_{0}^{1}$ band, has been accurately modelled as a rigid prolate symmetric top with first-order Coriolis coupling, analogous to the corresponding bands of benzene [35].

The quality of the fit is demonstrated in Fig. 9, which compares the R-branch region of the experimental and calculated spectrum. The appearance of weaker additional transitions, not predicted in the calculated symmetric top spectrum, suggests an internal rotation in the complex. The additional fact that the fitted $A_{v}^{\prime}(z)$ rotational constant is identical to the rotational constant $C_{v}^{\prime}(z)$ of free benzene, indicates a parallel-stacked structure in which the benzene behaves like the frame and the $\mathrm{N}_{2}$ can freely rotate about the benzene's $\mathrm{C}_{6}$ axis (see Fig. 10). An $A$ constant equal to that of the frame (where the $a$ inertial axis is aligned with the internal rotation axis), rather than that expected from a structural model, is a signature of sixfold internal rotation about this axis, as observed for example, for $\mathrm{CH}_{3} \mathrm{NO}_{2}$ and $\mathrm{C}_{6} \mathrm{H}_{6} \cdot \mathrm{SO}_{2}[31,36]$.

The accurately fitted $B$ rotational constants, on the other hand, are not contaminated by internal rotation since the

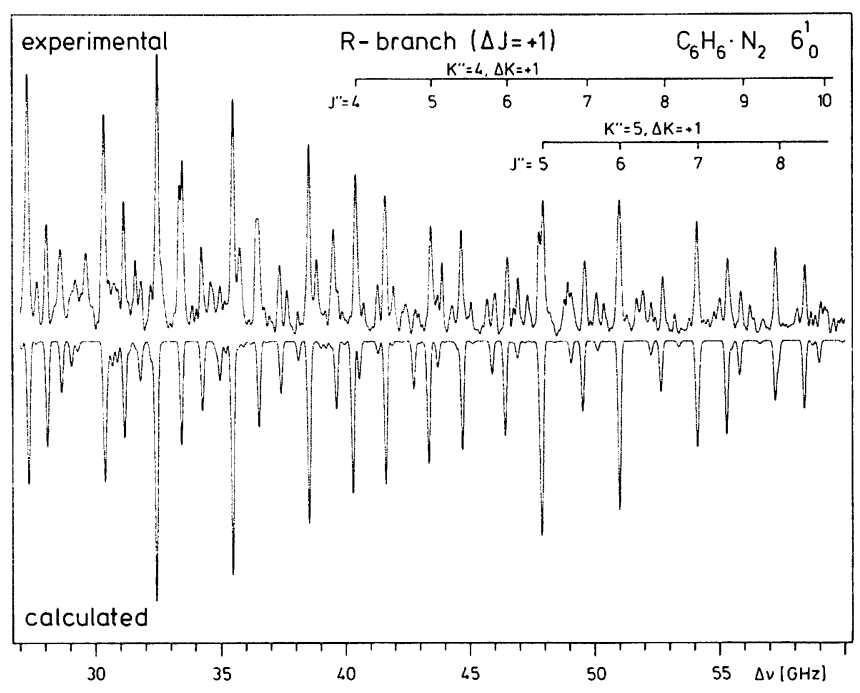

Fig. 9

Experimental and calculated spectrum of a part (R-branch) of the 60 band in $\mathrm{C}_{6} \mathrm{H}_{6} \cdot \mathrm{N}_{2}$. In the spectrum calculated from the fitted rotational constants of a symmetric top all strong experimental lines are reproduced. For explanation of the additional weaker lines in the experimental spectrum, see text (from Ref. [32]) 
internal rotation axis is perpendicular to the $B$ axis, i. e. these are the $B$ rigid rotor constants of the complex reflecting its structure. In fact, the $R_{\mathrm{cm}}$ 's (the distance between the center of mass of $\mathrm{N}_{2}$ and that of benzene) are uniquely determined from the accurately fitted $B$ rotational constants $\left(B_{0}^{\prime \prime}=\right.$ $0.048732(17) \mathrm{cm}^{-1}$ and $\left.B_{v}^{\prime}=0.049105(18) \mathrm{cm}^{-1}\right)$. These two van der Waals bond lengths are illustrated alongside the determined structure in Fig. 10. As for the benzene-noble gas dimers, the van der Waals bond distance decreases in the excited electronic state. They lie between that of $\mathrm{Ne}$ and $\mathrm{Ar}$, as one would expect naively from their mass numbers. The accuracy of the measured $R_{\mathrm{cm}}$ 's for benzene- $\mathrm{N}_{2}$ has been confirmed by measurement of the $6_{0}^{1}$ band of benzene- ${ }^{15} \mathrm{~N}_{2}$, as well as through the recent Fourier-transform microwave results for the ground electronic state of benzene- ${ }^{15} \mathrm{~N}_{2}[33]$.

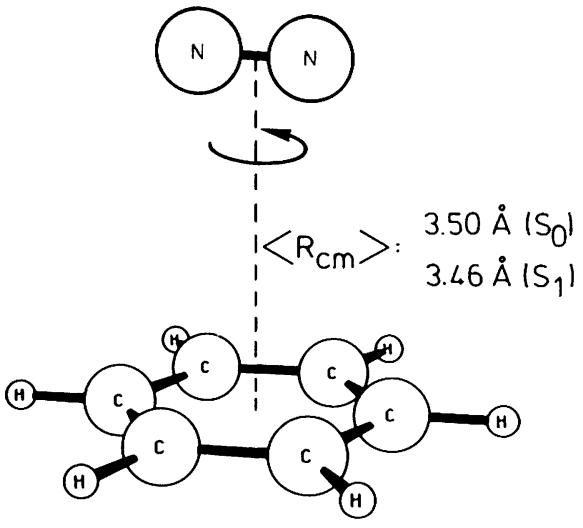

Fig. 10

Experimentally determined structure of the benzenc- $\mathrm{N}_{2}$ complex

To summarize, the rotationally resolved UV spectrum of the benzene- $\mathrm{N}_{2}$ complex shows that this complex is nonrigid with an internal rotation between the benzene and the nitrogen part of the complex. The internal rotation might be slightly hindered through the varying electron density along the benzene ring. Resolution of rotational-contorsional structure in the electronic spectrum allows a determination of the potential barriers for internal rotation in both the ground and excited electronic states. This opens up the interesting possibility of probing the effect of the electronic nodal structure of $S_{1}$ benzene on the solvent-solute interaction.

\section{Summary and Conclusion}

In this work we have demonstrated that rotationally resolved UV spectroscopy of complexes yields precise information on the structure and rigidity of complexes. For the prototype systems of benzene with noble gases or small molecules we found precise van der Waals distances. Rigid structures with sharp rotational lines have been detected not only for benzene-noble gas dimers but also for trimers with two Ar atoms attached to the benzene ring. When small diatomic molecules are attached to the benzene ring the complex is nonrigid due to the internal rotation of the molecule around the symmetry axis. Rotationally resolved spec- troscopy of van der Waals vibronic bands permits their unambiguous assignment. The vibrational energy and vibrationally averaged rotational constants provide information on the depth and the shape of the intermolecular potential essential for the modelling of physical and chemical properties of these prototype complexes.

The authors thank the Deutsche Forschungsgemeinschaft and the Fonds der Chemischen Industrie for financial support. They are indebted to Professor E. W. Schlag for his permanent interest in this work. A.M.S. acknowledges support of her work from the North Atlantic Treaty Organization in the form of a Postdoctoral Fellowship.

\section{References}

[1] D. H. Levy, Annu. Rev. Phys. Chem. 31, 197 (1980).

[2] For a review see: G. Scoles, "Atomic and Molecular Beam Methods", Vol. I, Oxford University Press, Oxford 1988.

[3] J. A. Beswick and J. Jortner, Adv. Chem. Phys. 47, 363 (1981).

[4] A. Amirav, U. Even, and J. Jortner, J. Chem. Phys. 75, 2489 (1981).

[5] E. R. Bernstein, K. Law, and M. Schauer, J. Chem. Phys. 80, 634 (1984).

[6] J. Bösinger and S. Leutwyler, Phys. Rev. Lett. 59, 1895 (1987).

[7] M. Y. Hahn and R. L. Whetten, Phys. Rev. Lett. 61, 1190 (1988).

[8] U. Even, N. Ben Horin, and J. Jortner, Chem. Phys.Lett. 156, 138 (1989).

[9] E. Riedle, H. J. Neusser, and E. W. Schlag, J. Chem. Phys. 75, 4231 (1981).

[10] W. Demtröder, Laser Spectroscopy, 3rd printing, chap. 10, Springer, Berlin 1988.

[11] C. A. Haynam, D. V. Brumbaugh, and D. H. Levy, J. Chem. Phys. 80, 2256 (1984).

[12] W. M. van Herpen and W. L. Meerts, Chem. Phys. Lett. 147, 7 (1988).

[13] A. Held and D. W. Pratt, J. Am. Chem. Soc. 112, 8629 (1990).

[14] Th. Weber, A. von Bargen, E. Riedle, and H. J. Neusser, J. Chem. Phys. 92, 90 (1990).

[15] K. H. Fung, H. L. Selzle, and E. W. Schlag, Z. Naturforsch. $36 a, 1338(1981)$

[16] J. A. Menapace and E. R. Bernstein, J. Phys. Chem. 91, 2533 (1987).

[17] Th. Weber, E. Riedle, H. J. Neusser, and E. W. Schlag, Chem. Phys. Lett. 183, 77 (1991); Th. Weber, E. Riedle, H. J. Neusser, and E. W. Schlag, J. Mol. Struct. 249, 69 (1991).

[18] S. Leutwyler, Chem. Phys. Lett. 107, 284 (1984).

[19] Th. Weber, doctoral thesis, Technische Universität München 1991.

[20] E. Riedle, Th. Weber, and H. J. Neusser, to be published.

[21] J. Jortner, U. Even, S. Leutwyler, and Z. Berkowitch-Yellin, J. Chem. Phys. 78, 309 (1983).

[22] H.-Y. Kim and M. W. Cole, J. Chem. Phys. 90, 6055 (1989).

[23] M. Mons and J. Le Calvé, Chem. Phys. 146, 195 (1990).

[24] G. Brocks and T. Huygen, J. Chem. Phys. 85, 3411 (1986).

[25] P. Hobza, H. L. Selzle, and E. W. Schlag, J. Chem. Phys. 95 , 391 (1991).

[26] B. Ernstberger, H. Krause, and H. J. Neusser, Z. Phys. D 20, 189 (1991).

[27] Th. Weber and H. J. Neusser, J. Chem. Phys. 94, 7689 (1991).

[28] M. Schmidt, M. Mons, and J. Le Calvé, Chem. Phys. Lett. 177, 371 (1991).

[29] M. Schmidt, M. Mons, J. Le Calvé, P. Millié, and C. CossartMagos, Chem. Phys. Lett. 183, 69 (1991).

[30] N. Ben-Horin, U. Even, and J. Jortner, Chem. Phys. Lett. 188, 73 (1992) 
[31] M. S. LaBarge, J. J. Oh, K. W. Hillig II, and R. L. Kuczkowski, Chem. Phys. Lett. 159, 559 (1989).

[32] Th. Weber, A. M. Smith, E. Riedle, H. J. Neusser, and E. W. Schlag, Chem. Phys. Lett. 175, 79 (1990).

[33] Y. Ohshima, H. Kohguchi, and Y. Endo, Chem. Phys. Lett. 184, 21 (1991).

[34] R. Nowak, J. A. Menapace, and E. R. Bernstein, J. Chem. Phys. 89, 1309 (1988).
[35] E. Riedle, Th. Knittel, Th. Weber, and H. J. Neusser, J. Chem. Phys. 91, 4555 (1989).

[36] C. C. Lin and J. D. Swalen, Rev. Mod. Phys. 31, 841 (1959).

Presented at the Discussion Meeting of the E 8092

Deutsche Bunsen-Gesellschaft für Physikalische Chemie "Reactions in and with Clusters" in Schliersee, Germany, March 29th - April 1st, 1992 